\title{
Redes de atenção à saúde: diagnostico das ações construídas entre a APS e os hospitais de pequeno porte
}

\author{
Lucas Gomes Costa de Paula
}

\section{RESUMO}

A busca por uma maior eficiência dos sistemas de saúde passa sobretudo pela organização dos serviços de saúde em redes de atenção. Estruturar os serviços de saúde, e assim garantir o atendimento integral e de qualidade para seus usuários, nas suas mais diversas complexidades e tendo como eixo coordenador a atenção primária a saúde tem sido o grande desafio. Este trabalho apresenta uma análise das relações existentes entre Hospitais com menos de $\mathbf{5 0}$ leitos e a Atenção Primária à Saúde no SUS.

Palavras-chave: Atenção Primária a Saúde; Redes de Atenção à Saúde, Hospitais de Pequeno Porte, Saúde Pública, Integralidade.

\section{ABSTRACT}

The search for greater efficiency in health systems depends mainly on the organization of health services in care networks. Structuring health services, and thus guaranteeing comprehensive and quality care for its users, in its most diverse complexities and having primary health care as its coordinating axis, has been the great challenge. This paper has as aim analysis of the relationships between Small Hospitals and Primary Health Care in National Health Care in Brazil.

Keywords: Primary Health Care; Health care networks; Small Hospitals; Public Health; Integrality
Revista da Rede APS 2021

Publicada em: 01/04/2021

DOI:10.14295/aps.v3i1.76

Lucas Gomes Costa de Paula (Hospital das Clínicas da Universidade Federal de Minas Gerais, Belo Horizonte, MG, Brasil)

Correspondência para:

Lucas Gomes Costa de Paula lucasgomes_cp@hotmail.com 


\section{INTRODUÇÃO}

Em relatório divulgado no início da década, a Organização Pan-Americana de Saúde (OPAS), alertou sua preocupação para o alto nível de fragmentação persistente nos sistemas de saúde da América Latina, destacando alguns dos principais problemas que dizem respeito a sua organização. Dificuldades no acesso, na disponibilidade dos serviços, na capacidade técnica, bem como o uso irracional e ineficiente dos recursos disponíveis, foram identificados como obstáculos para efetivação de um sistema integrado de atenção à saúde (1).

Longe de ser apenas um problema dos países latino-americanos, a crise contemporânea observadas nos grandes sistemas de saúde, é tida principalmente como resultado da fragmentação e segmentação de seus serviços, reflexo de um desencontro entre uma situação epidemiológica dominada por doenças crônicas e uma resposta social ineficaz, que não garante a continuidade dos processos assistenciais (2). No caso brasileiro, embora se tenha ao longo dos anos resultados expressivos para Sistema Único de Saúde (SUS) existe uma lacuna no que se refere ao planejamento e gestão, em especial à integralidade das ações, que enquanto princípio doutrinário do SUS, somente seria qualificada a partir da estruturação das redes de atenção (3).

Neste contexto, a proposta de organização do SUS em Redes de Atenção à Saúde (RAS) tem sido defendida devido aos ganhos de qualidade e eficiência, buscando romper o paradigma hospitalocêntrico centrado no cuidado do médico, para um modelo alternativo, que tenha a família e a comunidade como eixo norteador da assistência à saúde (4), atuando como um instrumento de garantia dos direitos, à medida que que se amplia o acesso e consequentemente diminui-se as desigualdades (5). Logo, garantir a integração da saúde, com qualificação e continuidade do cuidado, superando lacunas assistenciais e buscando a racionalização e otimização dos recursos disponíveis torna-se uma condição indispensável das redes regionalizadas e integradas da atenção (6).
Não obstante, a opção por um sistema público e universal, organizado de forma regionalizada tem gerado concomitantemente um duplo desafio: ofertar um conjunto de serviços a grupos majoritariamente desassistidos, sob o prisma da focalização de programas, como é observado na Estratégia de Saúde da Família (ESF) e, paralelamente, implantar as redes de atenção à saúde que, de fato, possam dar conta das necessidades de saúde da população (7). Sob este olhar, é preciso cuidado para não reforçar ainda mais o caráter fragmentado e segmentado do sistema, visto que a focalização da Atenção Primária à Saúde (APS), restritos a um determinado grupo populacional e constituído por um pacote básico de serviços, acaba sendo implementado em detrimento do acesso a níveis de maior complexidade (6).

Nesse sentido, posição semelhante ocupam também os serviços de média e alta complexidade. Tendo como amparo legal o princípio da integralidade, a assistência à saúde ao usuário do SUS também é garantida nos níveis de atenção em que o acesso se justifica pela necessidade de utilização de serviços mais densos tecnologicamente, não ofertados na atenção básica (AB). E, mesmo que a APS seja o eixo orientador do sistema e porta de entrada (preferencialmente) do usuário, por vezes, os procedimentos demandados por eles exigem maior grau de especialização e uso de tecnologias mais avançadas, na maioria das vezes, dispostas apenas nos hospitais.

A luz desta discussão e, considerando a estruturação das Redes de Atenção à Saúde, este artigo propõe analisar as ações estabelecidas entre a atenção primária a saúde e os Hospitais de Pequeno Porte do SUS.

\section{METODOLOGIA}

Este trabalho, adotou como estratégia metodológica a análise de dados obtidos a partir do survey censitário proveniente do projeto denominado como "Estudo de Hospitais de Pequeno Porte Brasileiros - Diagnóstico, Avaliação e Especialização" realizado por Facchini e Barbosa (8). Foi financiado pelo Ministério da Saúde e coordenado pela 
Universidade Federal de Minas Gerais via Observatório de Recursos Humanos em Saúde da Faculdade de Ciências Econômicas da UFMG em parceira com a Universidade Federal de Pelotas (UFPEL).

Neste artigo, especificamente, as variáveis analisadas contemplam as dimensões da APS, investigadas no instrumento. Dessa forma, para atender o propósito do estudo foram identificadas ações estabelecidas entre os Hospitais de Pequeno Porte (HPPs) e atenção básica (Tabelas 1, 2 e 3), cujas informações foram cruzadas com a finalidade de encontrar conexões existentes entre estes serviços. A expectativa com este trabalho é diagnosticar, levando-se em conta o papel central da APS nas RAS, possíveis evidências do fortalecimento das redes a partir das relações construídas entre esses dois níveis de atenção.

\section{RESULTADOS}

Os resultados expressados na Tabela 1 sugerem, em linhas gerais, que os HPPs apresentaram interações significativas junto a atenção primária em seus munícipios. Esse valor foi maior para as regiões Nordeste $(86,69 \%)$ e Sul $(87,21 \%)$. Não por menos estas duas regiões geográficas apresentavam em 2013, os maiores índices de cobertura da atenção básica dentre as demais $(81,6 \%$ e $73,05 \%)$ respectivamente (9). Do ponto de vista da natureza jurídica destes hospitais identificou-se um elevado nível de atuação em conjunto a $A B$ entre as instituições públicas e privadas s/fins lucrativos (Entre $78 \mathrm{e}$ $94 \%)$. Por outro lado, considerando os hospitais privados com fins lucrativos, apenas $1 / 3$ deles mantinham ações junto a $A B$.

Tabela 1 - Percentual dos HPPs que atuam de forma conjunta com a atenção básica e compartilha ações de educação permanente

\begin{tabular}{|c|c|c|c|c|c|c|c|c|}
\hline & \multicolumn{4}{|c|}{$\begin{array}{l}\text { O hospital atua de forma } \\
\text { conjunta com a } A B \text { ? }\end{array}$} & \multicolumn{4}{|c|}{$\begin{array}{l}\text { Há compartilhamento de ações } \\
\text { de educação permanente do } \\
\text { hospital com a AB? }\end{array}$} \\
\hline & \multicolumn{2}{|l|}{ Sim } & \multicolumn{2}{|l|}{ Não } & \multicolumn{2}{|l|}{ Sim } & \multicolumn{2}{|l|}{ Não } \\
\hline & $\mathbf{n}$ & $\%$ & $\mathbf{n}$ & $\%$ & $\mathbf{n}$ & $\%$ & $\mathbf{n}$ & $\%$ \\
\hline \multicolumn{9}{|l|}{ Região } \\
\hline Centro Oeste & 220 & 73,09 & 81 & 26,91 & 109 & 36,09 & 193 & 63,91 \\
\hline Nordeste & 749 & 86,69 & 115 & 13,31 & 356 & 41,25 & 507 & 58,75 \\
\hline Norte & 149 & 71,98 & 58 & 28,02 & 64 & 30,48 & 146 & 69,52 \\
\hline Sudeste & 356 & 78,41 & 98 & 21,59 & 148 & 32,89 & 302 & 67,11 \\
\hline Sul & 266 & 87,21 & 39 & 12,79 & 107 & 35,08 & 198 & 64,92 \\
\hline \multicolumn{9}{|l|}{ Natureza Jurídica } \\
\hline Público Federal & 16 & 84,21 & 3 & 15,79 & 9 & 47,37 & 10 & 52,63 \\
\hline Público Estadual & 130 & 78,31 & 36 & 21,69 & 61 & 36,97 & 104 & 63,03 \\
\hline Público Municipal & 1122 & 93,27 & 81 & 6,73 & 530 & 43,98 & 675 & 56,02 \\
\hline Privado s/ fins lucrativos & 373 & 84,77 & 67 & 15,23 & 147 & 33,49 & 292 & 66,51 \\
\hline Privado $\mathrm{c} /$ fins lucrativos & 96 & 32,65 & 198 & 67,35 & 36 & 12,29 & 257 & 87,71 \\
\hline \multicolumn{9}{|l|}{ Número de Leitos } \\
\hline 00 a 19 leitos & 512 & 85,05 & 90 & 14,95 & 246 & 40,73 & 358 & 59,27 \\
\hline 20 a 29 leitos & 489 & 81,64 & 110 & 18,36 & 217 & 36,11 & 384 & 63,89 \\
\hline 30 a 39 leitos & 408 & 80,79 & 97 & 19,21 & 170 & 33,86 & 332 & 66,14 \\
\hline 40 a 49 leitos & 209 & 79,47 & 54 & 20,53 & 80 & 30,77 & 180 & 69,23 \\
\hline $50+$ leitos & 122 & 75,31 & 40 & 24,69 & 71 & 43,56 & 92 & 56,44 \\
\hline \multicolumn{9}{|l|}{ Categoria } \\
\hline Hospital Geral & 1414 & 86,64 & 218 & 13,36 & 626 & 38,38 & 1005 & 61,62 \\
\hline Hospital Geral c/especialidade & 258 & 68,62 & 118 & 31,38 & 125 & 33,33 & 250 & 66,67 \\
\hline
\end{tabular}




\begin{tabular}{|c|c|c|c|c|c|c|c|c|}
\hline Hospital Especializado & 60 & 53,57 & 52 & 46,43 & 29 & 25,66 & 84 & 74,34 \\
\hline \multicolumn{9}{|l|}{ Quem arca com os custos do hospital? } \\
\hline Gestão Municipal & 1057 & 91,67 & 96 & 8,33 & 484 & 41,90 & 671 & 58,10 \\
\hline Gestão Estadual & 126 & 76,36 & 39 & 23,64 & 55 & 33,54 & 109 & 66,46 \\
\hline Gestão Municipal e Estadual & 330 & 92,70 & 26 & 7,30 & 161 & 45,48 & 193 & 54,52 \\
\hline Gestão Privada & 13 & 9,15 & 129 & 90,85 & 12 & 8,45 & 130 & 91,55 \\
\hline Gestão Federal & 8 & 72,73 & 3 & 27,27 & 4 & 36,36 & 7 & 63,64 \\
\hline \multicolumn{9}{|l|}{ Hospital contratualizado com o SUS? } \\
\hline Sim & 1430 & 87,20 & 210 & 12,80 & 658 & 40,15 & 981 & 59,85 \\
\hline Não & 185 & 53,01 & 164 & 46,99 & 75 & 21,43 & 275 & 78,57 \\
\hline \multicolumn{9}{|l|}{ População do Munícipio } \\
\hline Menor que 10 mil habitantes & 509 & 91,55 & 47 & 8,45 & 242 & 43,53 & 314 & 56,47 \\
\hline Entre $10 \mathrm{mil}$ e $50 \mathrm{mil}$ habitantes & 1052 & 84,70 & 190 & 15,30 & 454 & 36,70 & 783 & 63,30 \\
\hline Entre $50 \mathrm{mil}$ e $100 \mathrm{mil}$ habitantes & 68 & 62,39 & 41 & 37,61 & 33 & 28,95 & 81 & 71,05 \\
\hline Entre $100 \mathrm{mil}$ e $250 \mathrm{mil}$ habitantes & 32 & 43,24 & 42 & 56,76 & 13 & 17,57 & 61 & 82,43 \\
\hline Entre $250 \mathrm{mil}$ e $500 \mathrm{mil}$ habitantes & 17 & 48,57 & 18 & 51,43 & 10 & 29,41 & 24 & 70,59 \\
\hline Mais de 500 mil habitantes & 62 & 53,91 & 53 & 46,09 & 32 & 27,83 & 83 & 72,17 \\
\hline
\end{tabular}

Fonte: Elaborado pelo autor.

Em se tratando do porte destes hospitais aparentemente os resultados indicaram que quanto maior o número de leitos menor sua relação com a atenção básica. No tocante a população do município em que se encontravam estes hospitais, chama atenção o fato de que quanto menor o município maior era a proporção dos HPPs que atuavam de forma conjunta com atenção básica. Este resultado traduz o reflexo característico do programa de saúde da família à medida que fica claro sua proposta de focalização.

Em relação ao compartilhamento de ações de educação permanente dos hospitais com os serviços de atenção básica ou demais pontos da rede, chama atenção o maior valor entre os HPPs com 50 ou mais leitos. Ainda que estes não se enquadrem no conceito de pequeno porte foram os que tiveram em termos proporcionais mais ações compartilhadas junto a AB. Todavia, como de se esperar, hospitais especializados com gestão privada e não contratualizados com o SUS alcançaram baixos índices de parceira de atividades de educação permanente se comparado aos seus pares. Novamente, os HPPs em municípios com menos de 10 mil habitantes apontaram maior interação junto aos serviços básicos de saúde.

Tabela 2 - Percentual dos HPPs que compartilham profissionais com a AB

\begin{tabular}{|c|c|c|c|c|c|c|c|c|}
\hline & \multicolumn{4}{|c|}{ Médicos } & \multicolumn{4}{|c|}{ Enfermeiros } \\
\hline & \multicolumn{2}{|c|}{ Sim } & \multicolumn{2}{|c|}{ Não } & \multicolumn{2}{|c|}{ Sim } & \multicolumn{2}{|c|}{ Não } \\
\hline & $\mathbf{n}$ & $\%$ & $\mathbf{n}$ & $\%$ & $\mathbf{n}$ & $\%$ & $\mathbf{n}$ & $\%$ \\
\hline \multicolumn{9}{|l|}{ Região } \\
\hline Centro Oeste & 178 & 58,75 & 125 & 41,25 & 100 & 32,79 & 205 & 67,21 \\
\hline Nordeste & 554 & 63,90 & 313 & 36,10 & 393 & 45,38 & 473 & 54,62 \\
\hline Norte & 103 & 49,28 & 106 & 50,72 & 54 & 25,71 & 156 & 74,29 \\
\hline Sudeste & 238 & 52,65 & 214 & 47,35 & 147 & 32,52 & 305 & 67,48 \\
\hline Sul & 227 & 74,67 & 77 & 25,33 & 98 & 32,03 & 208 & 67,97 \\
\hline \multicolumn{9}{|l|}{ Natureza Jurídica } \\
\hline Público Federal & 12 & 63,16 & 7 & 36,84 & 8 & 42,11 & 11 & 57,89 \\
\hline Público Estadual & 83 & 50,00 & 83 & 50,00 & 57 & 34,13 & 110 & 65,87 \\
\hline
\end{tabular}




Público Municipal
Privado s/ fins lucrativos
Privado c/ fins lucrativos
Número de Leitos
00 a 19 leitos
20 a 29 leitos
30 a 39 leitos
40 a 49 leitos
$50+$ leitos
Categoria
Hospital Geral
Hospital Geral c/especialidade
Hospital Especializado
Quem arca com os custos do hospit

Quem arca com os custos do hospital?

Gestão Municipal

Gestão Municipal e Estadual

Gestão Privada

Gestão Federal

Hospital contratualizado com o SUS?

Sim

Não

População do Munícipio

Menor que 10mil habitantes

Entre $10 \mathrm{mil}$ e $50 \mathrm{mil}$ habitantes

Entre 50mil e $100 \mathrm{mil}$ habitantes

Entre $100 \mathrm{mil}$ e $250 \mathrm{mil}$ habitantes

Entre $250 \mathrm{mil}$ e $500 \mathrm{mil}$ habitantes

Mais de 500mil habitantes
Gestão Estadual

\begin{tabular}{|c|c|c|c|c|c|c|c|}
\hline 853 & 70,67 & 354 & 29,33 & 526 & 43,51 & 683 & 56,49 \\
\hline 270 & 61,36 & 170 & 38,64 & 153 & 34,85 & 286 & 65,15 \\
\hline 77 & 26,19 & 217 & 73,81 & 46 & 15,54 & 250 & 84,46 \\
\hline 384 & 63,37 & 222 & 36,63 & 245 & 40,43 & 361 & 59,57 \\
\hline 396 & 66,00 & 204 & 34,00 & 231 & 38,37 & 371 & 61,63 \\
\hline 289 & 57,46 & 214 & 42,54 & 181 & 35,91 & 323 & 64,09 \\
\hline 141 & 53,82 & 121 & 46,18 & 78 & 29,66 & 185 & 70,34 \\
\hline 90 & 54,88 & 74 & 45,12 & 57 & 34,76 & 107 & 65,24 \\
\hline 1054 & 64,46 & 581 & 35,54 & 626 & 38,17 & 1014 & 61,83 \\
\hline 201 & 53,46 & 175 & 46,54 & 130 & 34,67 & 245 & 65,33 \\
\hline 38 & 33,63 & 75 & 66,37 & 83 & 73,45 & 30 & 26,55 \\
\hline 801 & 69,23 & 356 & 30,77 & 495 & 42,71 & 664 & 57,29 \\
\hline 79 & 47,88 & 86 & 52,12 & 50 & 30,30 & 115 & 69,70 \\
\hline 252 & 70,79 & 104 & 29,21 & 151 & 42,42 & 205 & 57,58 \\
\hline 16 & 11,35 & 125 & 88,65 & 13 & 9,15 & 129 & 90,85 \\
\hline 5 & 45,45 & 6 & 54,55 & 2 & 18,18 & 9 & 81,82 \\
\hline 1082 & 65,82 & 562 & 34,18 & 657 & 39,89 & 990 & 60,11 \\
\hline 134 & 38,51 & 214 & 61,49 & 83 & 23,78 & 266 & 76,22 \\
\hline 428 & 76,43 & 132 & 23,57 & 234 & 41,86 & 325 & 58,14 \\
\hline 773 & 62,44 & 465 & 37,56 & 476 & 38,33 & 766 & 61,67 \\
\hline 46 & 40,71 & 67 & 59,29 & 32 & 28,07 & 82 & 71,93 \\
\hline 21 & 28,38 & 53 & 71,62 & 20 & 27,03 & 54 & 72,97 \\
\hline 10 & 28,57 & 25 & 71,43 & 9 & 25,71 & 26 & 74,29 \\
\hline 22 & 19,13 & 93 & 80,87 & 21 & 18,26 & 94 & 81,74 \\
\hline
\end{tabular}

Fonte: Elaborado pelo autor

De acordo com a Tabela 2, constatamos que os profissionais médicos que trabalham no HPPs possuem uma atuação maior junto $a A B$ do que os demais profissionais. Estende-se por este resultado que a escassez de médicos no município onde estão localizados estes hospitais, pode significar que este mesmo profissional também atua nos serviços de atenção básica. A possibilidade de trabalho com carga horaria reduzida nos hospitais, muito comum entre a classe médica, indica em maiores possibilidades de atuarem também sob outros vínculos empregatícios, tais como na $A B$.

Analisando sua natureza jurídica, destacam-se os hospitais públicos municipais e os federais, onde o compartilhamento de profissionais foi maior nessas instituições. Enquanto $70 \%$ dos hospitais públicos municipais compartilhavam médicos esse valor caia para em torno de $26 \%$ quando o HPP era privado com fins lucrativos. No caso dos enfermeiros a o percentual foi de $44 \%$ e $15 \%$ respectivamente. A mesma lógica ocorreu para o porte dos municípios, quanto maior, menor o compartilhamento de profissionais.

Hospitais contratualizados com o SUS assim como aqueles cuja gestão é pública se comparados aos hospitais não-contratuliazados ou que possuem a gestão privada o índice de compartilhamento de profissionais fui muito superior. É o que mostra o compartilhamento dos enfermeiros: $42 \%$, dos HPPs de Gestão Municipal e Estadual compartilhavam seus profissionais enquanto somente $9,15 \%$ dos HPPs de Gestão Privada tinham está prática. 
Tabela 3 - Percentual dos HPPs que participam do fórum de reunião com a atenção básica e tem suas consultas ambulatoriais também marcadas pela Unidade Básica de Saúde

\begin{tabular}{|c|c|c|c|c|c|c|c|c|}
\hline & \multicolumn{4}{|c|}{$\begin{array}{l}\text { O Hospital tem participação no } \\
\text { fórum de reunião com a } A B \text { ? }\end{array}$} & \multicolumn{4}{|c|}{ Marcação direta na UBS } \\
\hline & \multicolumn{2}{|l|}{ Sim } & \multicolumn{2}{|l|}{ Não } & \multicolumn{2}{|l|}{ Sim } & \multicolumn{2}{|l|}{ Não } \\
\hline & $\mathbf{n}$ & $\%$ & $\mathbf{n}$ & $\%$ & $\mathbf{n}$ & $\%$ & $\mathbf{n}$ & $\%$ \\
\hline \multicolumn{9}{|l|}{ Região } \\
\hline Centro Oeste & 70 & 67,96 & 33 & 32,04 & 61 & 20,89 & 231 & 79,11 \\
\hline Nordeste & 257 & 65,39 & 136 & 34,61 & 171 & 20,68 & 656 & 79,32 \\
\hline Norte & 54 & 64,29 & 30 & 35,71 & 18 & 9,38 & 174 & 90,63 \\
\hline Sudeste & 140 & 70,00 & 60 & 30,00 & 97 & 23,37 & 318 & 76,63 \\
\hline Sul & 84 & 67,74 & 40 & 32,26 & 78 & 26,90 & 212 & 73,10 \\
\hline \multicolumn{9}{|l|}{ Natureza Jurídica } \\
\hline Público Federal & 7 & 77,78 & 2 & 22,22 & 5 & 26,32 & 14 & 73,68 \\
\hline Público Estadual & 51 & 54,84 & 42 & 45,16 & 20 & 13,61 & 127 & 86,39 \\
\hline Público Municipal & 403 & 72,48 & 153 & 27,52 & 282 & 24,39 & 874 & 75,61 \\
\hline Privado s/ fins lucrativos & 125 & 60,68 & 81 & 39,32 & 97 & 23,89 & 309 & 76,11 \\
\hline Privado c/ fins lucrativos & 18 & 47,37 & 20 & 52,63 & 21 & 7,53 & 258 & 92,47 \\
\hline \multicolumn{9}{|l|}{ Número de Leitos } \\
\hline 00 a 19 leitos & 167 & 66,80 & 83 & 33,20 & 114 & 19,52 & 470 & 80,48 \\
\hline 20 a 29 leitos & 171 & 68,13 & 80 & 31,87 & 115 & 20,14 & 456 & 79,86 \\
\hline 30 a 39 leitos & 142 & 67,62 & 68 & 32,38 & 110 & 23,81 & 352 & 76,19 \\
\hline 40 a 49 leitos & 74 & 65,49 & 39 & 34,51 & 44 & 17,89 & 202 & 82,11 \\
\hline $50+$ leitos & 51 & 63,75 & 29 & 36,25 & 42 & 27,45 & 111 & 72,55 \\
\hline \multicolumn{9}{|l|}{ Categoria } \\
\hline Hospital Geral & 495 & 67,44 & 239 & 32,56 & 319 & 20,74 & 1219 & 79,26 \\
\hline Hospital Geral c/especialidade & 93 & 67,88 & 44 & 32,12 & 84 & 23,14 & 279 & 76,86 \\
\hline Hospital Especializado & 16 & 51,61 & 15 & 48,39 & 18 & 17,14 & 87 & 82,86 \\
\hline \multicolumn{9}{|l|}{ Quem arca com os custos do hospital? } \\
\hline Gestão Municipal & 377 & 70,34 & 159 & 29,66 & 267 & 24,23 & 835 & 75,77 \\
\hline Gestão Estadual & 40 & 48,78 & 42 & 51,22 & 18 & 12,24 & 129 & 87,76 \\
\hline Gestão Municipal e Estadual & 129 & 76,33 & 40 & 23,67 & 91 & 27,08 & 245 & 72,92 \\
\hline Gestão Privada & 1 & 16,67 & 5 & 83,33 & 2 & 1,49 & 132 & 98,51 \\
\hline Gestão Federal & 1 & 50,00 & 1 & 50,00 & 3 & 27,27 & 8 & 72,73 \\
\hline \multicolumn{9}{|l|}{ Hospital contratualizado com o SUS? } \\
\hline Sim & 479 & 65,62 & 251 & 34,38 & 354 & 22,90 & 1192 & 77,10 \\
\hline Não & 70 & 66,67 & 35 & 33,33 & 45 & 13,60 & 286 & 86,40 \\
\hline \multicolumn{9}{|l|}{ População do Munícipio } \\
\hline Menor que 10 mil habitantes & 179 & 74,58 & 61 & 25,42 & 128 & 23,88 & 408 & 76,12 \\
\hline Entre $10 \mathrm{mil}$ e $50 \mathrm{mil}$ habitantes & 369 & 64,96 & 199 & 35,04 & 253 & 21,53 & 922 & 78,47 \\
\hline Entre $50 \mathrm{mil}$ e $100 \mathrm{mil}$ habitantes & 27 & 62,79 & 16 & 37,21 & 18 & 17,65 & 84 & 82,35 \\
\hline Entre $100 \mathrm{mil}$ e $250 \mathrm{mil}$ habitantes & 9 & 42,86 & 12 & 57,14 & 10 & 15,15 & 56 & 84,85 \\
\hline Entre $250 \mathrm{mil}$ e $500 \mathrm{mil}$ habitantes & 5 & 71,43 & 2 & 28,57 & 3 & 9,38 & 29 & 90,63 \\
\hline Mais de 500 mil habitantes & 16 & 64,00 & 9 & 36,00 & 13 & 12,38 & 92 & 87,62 \\
\hline
\end{tabular}

Fonte: Elaborado pelo autor 
Retomando um pouco aos dados coletados no inquérito original, que buscou evidenciar a existência de conselhos assistenciais nos HPPs, nota-se que dentre aqueles que hospitais que afirmaram participar das instancias de governança aproximadamente $67 \%$ deles diziam ter participação nos fóruns de reunião com a atenção básica. Esse valor é ainda maior proporcionalmente, olhando a Tabela 3, nos HPPs da Região Sudeste (70\%); entre aqueles de Natureza Jurídica Federal $(77,78 \%)$ e Municipal (72,48\%); nos HPPs de 20 a 29 leitos (68,13\%); nos Hospitais Gerais $(67,44 \%)$; hospitais com os custos arcados pelo município e estado (76,33\%); entre os não contratualizados com o SUS $(66,67 \%)$ e os localizados em municípios com menor que 10 mil habitantes (74,58\%).

Quanto ao nível de atuação do HPP sob regulação foi perguntado se a marcação de consultas ambulatoriais era também realizada via Unidade Básica de Saúde (UBS). A região Sul tinha $27 \%$ dos seus hospitais com menos de 50 leitos com marcação realizada também na UBS. Dos hospitais privados com fins lucrativos e que provavelmente também atendem SUS este valor foi $7 \%$, ao contrário dos hospitais municipais na qual $1 / 4$ deles tinham consultas marcadas no posto de saúde. Resultado semelhante ocorre nos municípios de até 50 mil habitantes cuja taxa de marcação via UBS foram as maiores entre os demais municípios com maior porte populacional.

\section{DISCUSSÃO}

Em se tratando dos HPPs, a ineficiência econômica, traduzida pela ausência de ganhos de escala e escopo, contrasta sobretudo na atuação estratégica que desempenham no sistema. Parte considerável destes hospitais se localiza fora dos grandes centros urbanos, o que demonstra seu poder de capilaridade para o interior do país, e ao mesmo tempo acirra os debates acerca da sua viabilidade.

Fato é, conforme este trabalho propôs apresentar, que os HPPs têm um papel a desempenhar no fortalecimento das redes de atenção. Obviamente agora mais do que nunca, no contexto das RAS, estes hospitais precisam servir como referência e contra referência da APS. E foi em linhas gerais o que os resultados apontaram, visto que $80 \%$ dos hospitais atuavam junto $a A$. $O$ que não necessariamente foi observado nos outros parâmetros tais como o de marcação das consultas pela UBS.

Todas as categorias de certa forma diziam muito sobre relação dos HPPs com a AB. Os índices de cobertura e de qualidade foram observados, principalmente nas regiões Nordeste e Sul. A natureza jurídica tanto quanto a gestão dos HPPs mostrou um certo grau de desigualdade ao realçar uma maior interação entre os serviços públicos e a atenção primária, e uma baixa vinculação na rede entre os serviços privados. Resultados semelhantes para os HPPs contratualizados com SUS, que em termos percentuais também tiveram maior conexão com os serviços da $A B$ se comparados com aqueles não contratualizados.

Por fim, do ponto de vista espacial, ficou ainda mais evidente a importância dos HPPs no fortalecimento da rede de atenção à saúde, ainda que local. Hospitais localizados em munícipios com menor número de habitantes, tinha interações maiores e compartilhavam mais os profissionais junto a atenção básica. $E$ muito embora estes hospitais estejam longe dos grandes centros, dos recursos tecnológicos, humanos e de formação, muitos se mantem como referência no atendimento à população local. Cabe aos gestores entender o papel que ali ocupam e por meio deles garantir a integralidade da atenção à saúde estreitando ainda mais seus vínculos com a atenção primária à saúde. 


\section{NOTAS E REFERÊNCIAS}

(1) OPAS - Organización Pan-Americana de la Salud. La Renovación de la Atención Primaria de Salud en las Américas. Redes Integradas de Servicios de Salud: Conceptos, Opciones de Política y Hojavde Ruta para su Implementación en las Américas. Washington DC: OPAS, 2010

(2) OPAS - Organização Pan-Americana de Saúde. A atenção à saúde coordenada pela APS: construindo as redes de atenção no SUS: contribuições para o debate. Brasília: Organização Pan-Americana da Saúde, 2011.

(3) Rivaldo Mauro de Faria. Atenção Primária, o Território, e as Redes de Atenção: intercambiamentos necessários para a integração das ações do Sistema Único de Saúde (SUS) em Minas Gerais. Revista Brasileira de Geografia Médica e da Saúde. Hygeia 10 (19): 8 - 23, Dez/2014.

(4) Eugênio Vilaça Mendes. As Redes de Atenção à Saúde. Brasília: Organização Pan-Americana da Saúde, 2011.

(5) Rosana Kuschnir e Adolfo Horácio Chorny. Redes de atenção à saúde: contextualizando o debate. Ciência \& Saúde Coletiva, 15(5):2307-2316, 2010.

(6) Silvio Fernandes da Silva. Organização das redes regionalizadas e integradas de atenção à saúde: desafios do Sistema Único de Saúde (Brasil). Ciência \& Saúde Coletiva, 16(6):2753-2762, 2011

(7) CONASS. Conselho Nacional de Secretários de Saúde. Assistência de média e alta complexidade. Brasília: CONASS, 2011.

(8) FACCHINI, L. A.; BARBOSA, A. C. Q. B (coord.). Estudo de Hospitais de Pequeno Porte Brasileiros diagnóstico, avaliação e espacialização. Belo Horizonte/Pelotas: UFPEL e Observatório de Recursos Humanos em Saúde da FACE/UFMG, 2014. (Relatório de pesquisa)

(9) Ministério da Saúde (MS). Secretaria de Atenção Primária à Saúde. 2013. Disponível em: https://egestorab.saude.gov.br/paginas/acessoPublico/relatorios/relHistoricoCoberturaAB.xhtml 